\title{
Tesla-Scale Terahertz Magnetic Impulses
}

\author{
Shawn Sederberg, Fanqi Kong, and Paul B. Corkum \\ Joint Attosecond Science Laboratory, University of Ottawa and National Research Council Canada, \\ 25 Templeton Street, Ottawa, Ontario K1N 7N9 Canada
}

(Received 6 September 2019; revised manuscript received 29 November 2019; accepted 12 February 2020; published 13 March 2020)

\begin{abstract}
Measuring the magnetic response of matter relies acutely on the degree to which a magnetic field source's amplitude, spatial, and temporal character can be tailored. Magnetic fields are inseparable from light-matter interaction, yet due to the dominance of electric-field-induced effects in many systems, laser pulses have heretofore provided comparatively limited insight into the high-frequency magnetic response of matter. Conductors or superconductors arranged in a solenoidal configuration embody the state-ofthe-art apparatus for generating spatially isolated magnetic fields, but the reliance on electrical circuitry limits the field amplitude, pulse brevity, and absolute timing of the generated fields. We transfer the concept of solenoidal currents commonly leveraged in electromagnets to photo-ionized electrons driven by moderately intense vector laser beams, in a scheme that does not require the laser mode to carry orbital angular momentum. We predict that this all-optical approach will enable magnetic fields exceeding 8 Tesla to be turned on within 50 femtoseconds using moderate laser intensities, an unprecedented combination of parameters that will open the possibility for ultrafast metrological techniques to be combined with intense, spatially isolated, magnetic fields.
\end{abstract}

DOI: 10.1103/PhysRevX.10.011063

Magnetic fields play a fundamental role in our understanding of magnetic materials [1], electron spins [2,3], superconductivity [4-6], quantum and topological systems [7-9], plasma and nuclear fusion [10-12], medical diagnosis [13], and astrophysics [14]. Electric charge in motion generates magnetic fields $[15,16]$, and the arrangement of conductors or superconductors into loops or solenoids has been the workhorse approach to magnetic field sources for decades. Although effective for measuring the quasistatic magnetic response of matter, the ultrafast dynamics that ultimately produces this response remains largely inaccessible.

As an alternative, relativistic electron bunches have been used to introduce a strong magnetic pulse of 2.3 ps duration to a granular recording film [17]. The application of large magnetic fields of short duration enables insight into the maximum switching bandwidth of the magnetic material before the onset of magnetic disorder. Although these results provide intriguing fundamental insight with important technological implications, the large-scale electron acceleration facility required for these measurements limits

\footnotetext{
*msederbe@uottawa.ca
}

Published by the American Physical Society under the terms of the Creative Commons Attribution 4.0 International license. Further distribution of this work must maintain attribution to the author(s) and the published article's title, journal citation, and DOI.
Subject Areas: Atomic and Molecular Physics, Magnetism, Optics

the exploration of ultrafast dynamics in other material systems.

Extensive effort has been devoted to optically exciting solenoidal currents and high magnetic fields in underdense plasmas using relativistic laser pulses, for the purpose of charged beam collimation. Early approaches were based on the inverse Faraday effect [18], whereby the angular momentum is transferred from a circularly polarized beam to the plasma via dissipative effects. The recent availability of optical vortex beams [i.e., laser modes that carry orbital angular momentum (OAM)] at relativistic intensities [19] has motivated new approaches to transfer angular momentum from the laser mode to a solenoidal plasma current. Wu et al. have demonstrated that when a weak magnetostatic seed is applied along the propagation axis of a vortex beam, net angular momentum is transferred to the plasma, amplifying the seed up to $10 \mathrm{kT}$ [20]. Nuter et al. proposed that the combination of strong radial and longitudinal electric fields present in tightly focused radial laser beams can transfer angular momentum to electrons through a dissipationless, optical process, resulting in 100-T magnetic fields [21]. Shi et al. have demonstrated that two copropagating vortex beams with different frequencies and topological charge produce a twisted ponderomotive force, leaving behind a solenoidal current and quasistatic magnetic field up to $40 \mathrm{~T}$ [22].

Although these would be powerful new techniques for charged beam collimation, they each rely on laser modes 
that contain OAM and intensities that are relativistic or near relativistic. The requirement for high intensities suggests that the light-plasma angular-momentum transfer is relatively inefficient in these schemes, and it also prohibits their use in university-scale laboratories. High efficiency transfer of energy from electric fields to a solenoidal current would reduce the required intensities and make its application relevant for many researchers in the field of ultrafast magnetism.

The magnetic field generated at the center of an ideal solenoid is expressed by the relation $B=\mu n I$, where $\mu$ is the permeability of the core material, $n=N / L$ is the number of wire turns per unit length, and $I$ is the circulating current. Generating large magnetic fields is, therefore, inseparable from confining high current to a compact volume. Momentum transfer from a laser field to electrons is perhaps the most direct and efficient means to generate high current densities and magnetic fields at subrelativistic intensities. Atoms subjected to sufficiently intense light give rise to photo-ionized electrons, each of which undergoes a unique trajectory, and the collective electron trajectories contribute to an overall current density. In the case of photo-ionization driven by a monochromatic laser pulse at angular frequency, $\omega$, electrons experience symmetric acceleration in the orientation of the field's polarization. In other words, the instants of ionization coincide with null points in the field's vector potential, yielding negligible net current density. The addition of a second harmonic field $2 \omega$, with the proper phase relative to the fundamental, $\Delta \varphi_{\omega, 2 \omega}$, breaks this symmetry and shifts the ionization bursts to nonzero instants in the vector potential, yielding a current density that persists until the onset of dephasing. Such asymmetric interaction with optical fields is a form of coherent control, and it enables sensitive control of the amplitude and direction of electrical currents in semiconductors and gases by the adjustment of $\Delta \varphi_{\omega, 2 \omega}[23-25]$.

A gas medium with a high ionization potential $I_{p}$ permits application of strong optical fields before the gas is ionized. Atomic helium $\left(I_{p}=24.6 \mathrm{eV}\right)$ can withstand electric fields exceeding $E_{0}=7 \mathrm{~V} / \AA$ (intensity, $I_{0}=6.5 \times 10^{14} \mathrm{~W} / \mathrm{cm}^{2}$ ). We consider two-color excitation using parameters that we will later apply to more detailed simulations: $\lambda=4000 \mathrm{~nm}$ pulses of $\tau_{p}=40 \mathrm{fs}$ duration and peak electric field strength $E_{\omega}=7.4 \mathrm{~V} / \AA$ $\left(I_{\omega}=7.3 \times 10^{14} \mathrm{~W} / \mathrm{cm}^{2}\right)$, and $\lambda=2000 \mathrm{~nm}$ pulses of $\tau_{p}=40$ fs duration and peak electric field strength $E_{2 \omega}=$ $2.3 \mathrm{~V} / \AA\left(I_{2 \omega}=0.70 \times 10^{14} \mathrm{~W} / \mathrm{cm}^{2}\right)$. Evaluating ionization and classical trajectories in the two-color field reveals that electrons are imparted with an average velocity $v=6.9 \times 10^{6} \mathrm{~m} / \mathrm{s}$. An ionized gas population on the order of $n_{i}=1.5 \times 10^{18} \mathrm{~cm}^{-3}$ imparted with this velocity would result in an optical-field-driven current density on the order of $|J|=|q| n_{i} v=165 \mathrm{MA} / \mathrm{cm}^{2}$. This result surpasses what is possible in conductors or superconductors by approximately 3 orders of magnitude. Harnessing photoexcited currents offers the possibility for ultrafast, optically synchronized, magnetic fields with amplitudes similar to those generated in state-of-the-art electromagnets.

By relaxing the requirement for a solenoid composed of wires, one can envisage a solenoid consisting of a continuous sheet of circulating charge. In this case, an equivalent expression for the ideal solenoid equation can be written as $B=\mu I_{L}$, where $I_{L}$ is the circulating current per unit length. This scenario can be realized using $\omega$ and $2 \omega$ azimuthal vector beams to control photo-ionization of atoms. The azimuthal electric field distribution is shown in Fig. 1(b), where the electric fields in the doughnut shape have linear polarization pointing in the azimuthal direction [26], and the beam does not carry OAM. Coherent control of photo-ionization transfers the spatial intensity and polarization configuration of the azimuthal beams to the collective electron motion of the ionized gas. The azimuthal motion of each photoionized electron in the entire beam can be grouped into an effective azimuthal current density $J_{\theta}$, and this current density induces a longitudinal magnetic field $B_{z}$. As with conventional coherent control, the direction and amplitude of both $J_{\theta}$ and $B_{z}$ can be precisely controlled by the adjustment of $\Delta \varphi_{\omega, 2 \omega}$.

Notably, this approach is unrelated to the OAM of the laser beam as it relies on directly transferring momentum from the local electric field of the laser beam to a local element of the solenoidal current. Using the same $\omega / 2 \omega$ pulse parameters as above, we can estimate how efficiently each absorbed photon is converted into electron angular momentum. The number of photons required to accelerate an electron to velocity $v$ can be written as

$$
N_{\text {phot }}=\frac{T \lambda}{h c}
$$

where $T$ is the kinetic energy of an electron, $c$ is the speed of light in vacuum, and $h$ is the Planck constant. The number of units of angular momentum can be expressed as

$$
N_{\mathrm{AM}}=\frac{m_{e} v r}{\hbar},
$$

where $m_{e}$ is the electron mass, $r$ is the radius from the beam center at which the electron is moving, and $\hbar$ is the reduced Planck constant. The units of angular momentum transferred to the solenoidal current per absorbed photon can be simplified to

$$
\frac{N_{\mathrm{AM}}}{N_{\text {phot }}}=\frac{4 \pi r c}{\lambda v} .
$$

Using $\lambda=4000 \mathrm{~nm}$ and $v=6.9 \times 10^{6} \mathrm{~m} / \mathrm{s}$, we consider an azimuthal laser beam that is focused to a $w_{0}=$ $100 \mu \mathrm{m}$ beam waist and find that electrons at $r=100 \mu \mathrm{m}$ 

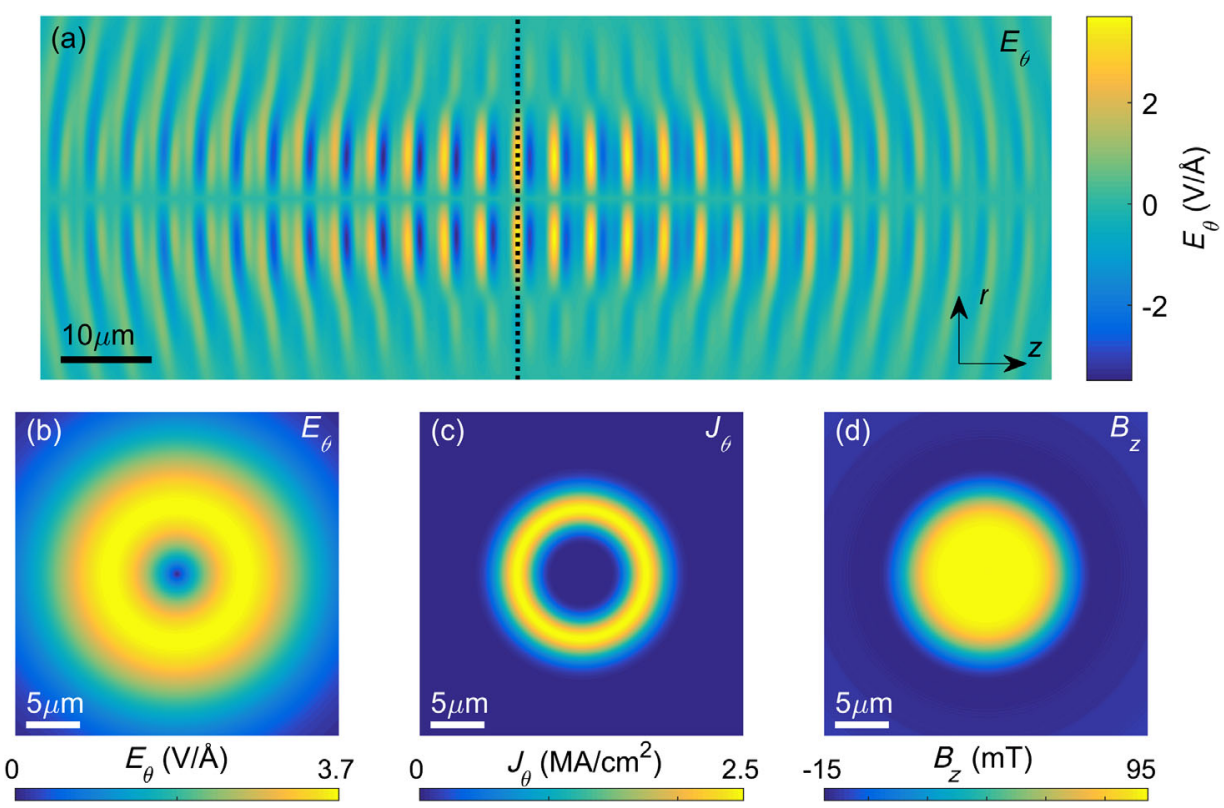

FIG. 1. Numerical simulations of strong field coherent control in atomic hydrogen using azimuthal vector beams. (a) A snapshot of the electric field from an azimuthal vector beam propagating through a Gaussian focusing geometry with beam waist, $w_{0}=6 \mu \mathrm{m}$. (b) A transverse slice of the azimuthal electric field mode at the beam waist. (c) A transverse slice of the azimuthal current density at the beam waist. (d) A transverse slice of the longitudinal magnetic field produced by the current density at the beam waist.

will receive $1.4 \times 10^{4}$ units of angular momentum per absorbed photon. We note that both momentum and angular momentum are conserved by giving an equal, but opposite momentum and angular momentum to the ions. Such an efficient intensity-driven solenoidal current holds the potential to reduce the pulse energies required for generating strong magnetic fields to those available from commercial laser systems.

For an azimuthal laser beam focused into helium gas to a $w_{0}=100 \mu \mathrm{m}$ beam waist, one can estimate confinement of the gas ionization to a radial extent of approximately $\Delta r=20 \mu \mathrm{m}$. The current per unit length can be calculated as $I_{L}=J_{\theta} \Delta r=3.3 \times 10^{7} \mathrm{~A} / \mathrm{m}$, which would produce magnetic fields on the order of $B_{z}=\mu_{0} I_{L}=41.4 \mathrm{~T}$, similar to what is produced by state-of-the-art magnetic field sources. However, turning on such high magnetic fields using femtosecond laser pulses would result in a large back electromotive force (called back-EMF here) acting on the ionized electrons. Faraday's law,

$$
\oint \overrightarrow{\boldsymbol{E}}_{\boldsymbol{E M F}} \cdot d \vec{\ell}=-\int \frac{\partial \overrightarrow{\boldsymbol{B}}}{\partial t} \cdot d \overrightarrow{\boldsymbol{A}}
$$

can be used to estimate the amplitude of the back-EMF field present at the beam radius, $w_{0}$ :

$$
2 \pi w_{0} E_{\mathrm{EMP}}=-\pi w_{0}^{2} \frac{\Delta B}{\Delta t} .
$$

Turning on $\Delta B=40 \mathrm{~T}$ within $\Delta t=50$ fs produces a back-EMF, $E_{\mathrm{EMF}}=-4 \mathrm{~V} / \AA$, which is similar in amplitude to the driving field. These estimates suggest that, while we may not reach $40 \mathrm{~T}$, large, rapid rise-time, isolated $B$ fields are feasible. To proceed, we need a model that can capture the complex spatiotemporal coupling of charge motion, induced magnetic fields, and back-EMF fields.

To account for these intricacies, we simulate this physical scenario using an approach that is closely related to particle-in-cell calculations [27]. We present details of the simulation technique in the Supplemental Materials [28]. We first consider a fundamental laser pulse at $\lambda_{\omega}=$ $4000 \mathrm{~nm}$ and its second harmonic at $\lambda_{2 \omega}=2000 \mathrm{~nm}$ focused into atomic hydrogen $\left(I_{p}=13.6 \mathrm{eV}, \quad n=\right.$ $10^{17} \mathrm{~cm}^{-3}$ ) to azimuthal electric field strengths of $E_{\theta, \omega}=$ $2.9 \mathrm{~V} / \AA\left(I_{\omega}=1.1 \times 10^{14} \mathrm{~W} / \mathrm{cm}^{2}\right)$ and $E_{\theta, 2 \omega}=1.2 \mathrm{~V} / \AA$ $\left(I_{2 \omega}=0.19 \times 10^{14} \mathrm{~W} / \mathrm{cm}^{2}\right)$, respectively; identical pulse durations of $\tau_{p}=40 \mathrm{fs}$; and a relative phase, $\Delta \varphi_{\omega, 2 \omega}=5.01 \mathrm{rad}$. Each pulse is focused to the same beam radius, $w_{0}=6 \mu \mathrm{m}$, with corresponding pulse energies of $11.3 \mu \mathrm{J}$ for the fundamental and $1.9 \mu \mathrm{J}$ for the second harmonic.

The two pulses are propagated through the Rayleigh length of a Gaussian focusing geometry, as depicted in Fig. 1(a). It should be noted that the Gouy phase shift produces a phase slip between the two pulses as they propagate. At each time step, the ionization rate is evaluated at every mesh point, and all existing electron trajectories are updated. The electron trajectories are distributed into a current density mapping, which is included in the next time step of Maxwell's equations for a self-consistent account of plasma backaction. Inclusion of plasma dispersion ensures 

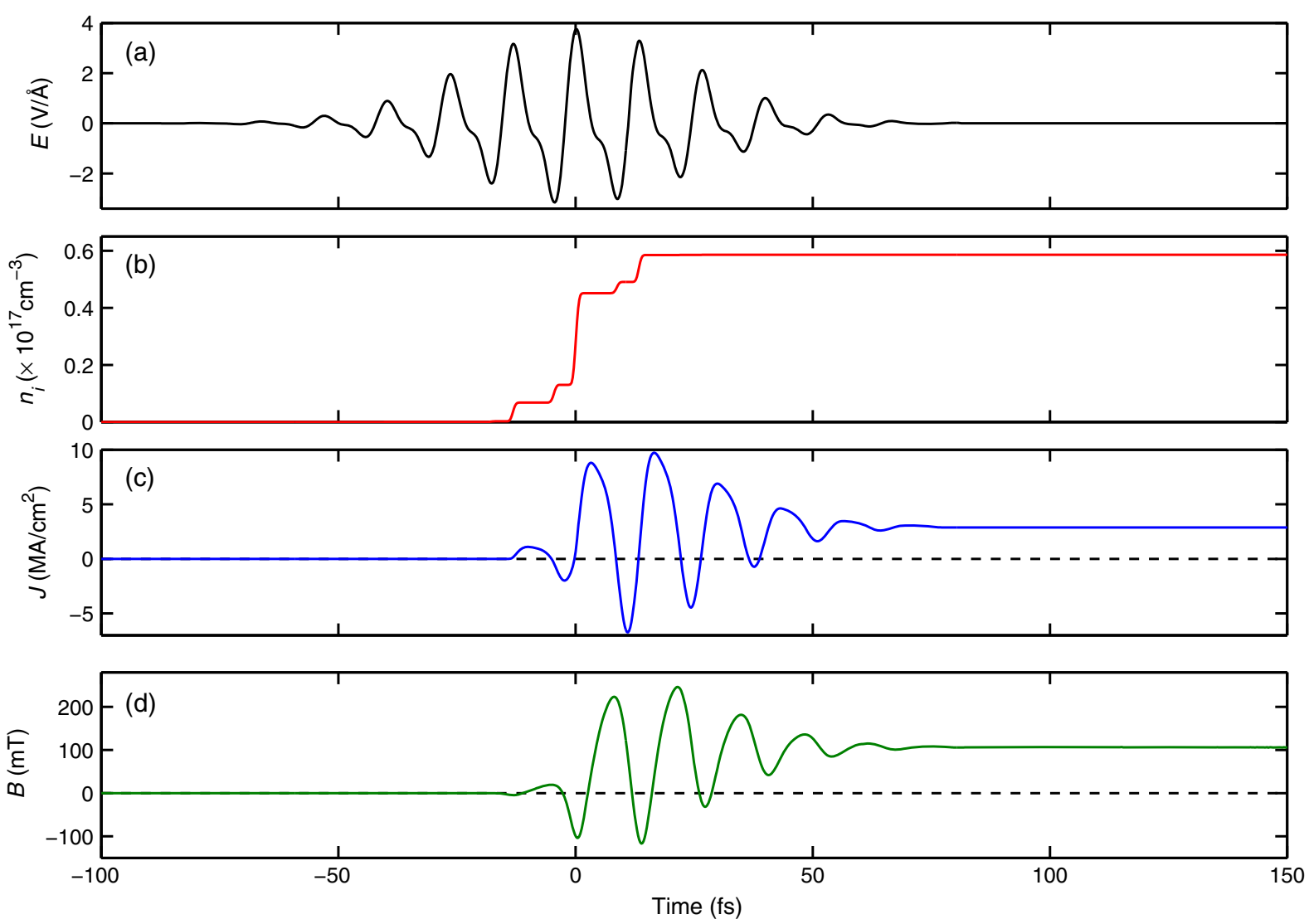

FIG. 2. Temporal dynamics relating the electric field excitation, gas ionization, current generation, and magnetic field induction. (a) An exemplary $\omega / 2 \omega$ electric field waveform at the beam waist. (b) Growth of the ionized population density when this field is incident on a hydrogen gas target with a density $n=10^{17} \mathrm{~cm}^{-3}$. (c) The current density dynamics that occurs as the excited population interacts with the remainder of the laser pulse. (d) The magnetic field produced by this current density.

accurate modeling of any additional phase slip between the two beams as they copropagate.

Figure 1(c) shows a transverse slice of the current density excited by the driving laser beams at the beam waist. The spatial intensity distribution of the azimuthal laser beam combined with the extreme nonlinearity of the photoionization process confines the excitation of an azimuthal current to a thin circular loop in the transverse plane. Electrons at each azimuthal position, $\theta_{i}$, are briefly given momentum in the azimuthal direction, producing a pulsed collective current that resembles a solenoidal current. This current induces and encompasses a spatially uniform longitudinal magnetic field $B_{z}$, as shown in Fig. 1(d).

The corresponding temporal dynamics giving rise to this solenoidal current and its accompanying magnetic field for the above results are presented in Fig. 2. The exciting electric field waveform at the beam waist is shown in Fig. 2(a). With a majority of the ionization occurring near or after the peak of the laser pulse, plotted in Fig. 2(b), the buildup of the current density primarily occurs in the latter half of the laser pulse, as depicted in Fig. 2(c). A magnetic field is induced by this current, and its dynamic buildup is presented in Fig. 2(d).
This magnetic field will exist for as long as the azimuthal current remains coherent.

Atmospheric density plasmas lose coherence on a picosecond timescale, and they have been shown to radiate terahertz (THz) bandwidth impulses, which propagate into the far field [29]. In the case of Gaussian laser beams, a comparison can be made to an electric dipole antenna radiating into the far field. By carefully collimating the strongly diverging $\mathrm{THz}$ pulse and applying a long pass filter to remove the optical frequency excitation light, the single-cycle or even subcycle terahertz pulse can be refocused onto the desired target.

The present case is analogous to a magnetic dipole antenna that radiates into the far field. Just as the spatial mode of the radiated $\mathrm{THz}$ pulse resulting from Gaussian beam excitation is essentially preserved, the propagating mode radiated from azimuthal beam excitation will be similar to an azimuthal beam. Because of the single-cycle duration of the $\mathrm{THz}$ pulse, the radiated transient will exhibit a space-time structure closely resembling a flying electromagnetic torus [30]. An azimuthal laser mode naturally contains a longitudinal magnetic field, and therefore, the 
solenoidal magnetic field transient will be present in the radiated $\mathrm{THz}$ pulse.

In a similar manner as $\mathrm{THz}$ pulses produced by Gaussian beams are routinely collimated, isolated, and focused using conventional optical techniques, we anticipate delivery of intense, spatially isolated magnetic fields to solid-state samples. The ability to generate and synchronize magnetic impulses with ultrafast optical characterization schemes would enable insight into the response of matter to magnetic fields using time-resolved techniques analogous to those pioneered by the $\mathrm{THz}$ and attosecond communities [31-35].

Adjusting $\Delta \varphi_{\omega, 2 \omega}$ imposes coherent control on the excited current density and the resulting magnetic fields. Figure 3(a) shows the peak magnetic field at the beam waist as $\Delta \varphi_{\omega, 2 \omega}$ is adjusted. The relative phase also strongly influences the spatial distribution of magnetic fields that are excited, and four slices taken in the longitudinal plane of the focusing geometry are shown in Figs. 3(b)-3(e) for $\Delta \varphi_{\omega, 2 \omega}=\pi / 8,5 \pi / 8,9 \pi / 8$, and $13 \pi / 8$, respectively. Notably, $\Delta \varphi_{\omega, 2 \omega}$ determines whether the longitudinal magnetic field closely resembles that of a solenoid, such as in Figs. 3(c) and 3(e), or that of two opposing magnetic dipoles, such as in Figs. 3(b) and 3(d). A superposition of these two cases is observed for the other $\Delta \varphi_{\omega, 2 \omega}$ values. It is important to note that the Gouy phase shift of an azimuthal laser beam is $\Delta \varphi_{\text {Gouy }}=2 \pi$, and this value influences the spatial distribution of the excited current density as the laser beams propagate through the focusing geometry.

Increasing $w_{0}$ while keeping the peak electric field constant (i.e., by increasing the laser pulse energy) produces a larger solenoidal current per unit length, and our calculations demonstrate a linear scaling relationship, i.e., $J_{L} \propto w_{0}$. Therefore, increasing the laser beam waist provides a simple and convenient means to scale the magnetic field amplitude. With the availability of energetic femtosecond laser pulses, an important question that must be addressed is as follows: To what magnetic field amplitude may this scheme be scaled before plasma backaction suppresses the excited current density and resulting magnetic field?

To assess this question, we increase the beam waist to $w_{0}=100 \mu \mathrm{m}$, which, according to a simple application of the ideal solenoid equation, would generate Tesla-scale magnetic fields in the absence of back-EMF. Pulses with the same parameters as stated previously are propagated through $L=200 \mu \mathrm{m}$ of atomic hydrogen. Notably, we maintain the same electric field strengths while increasing the focal spot size, which would require $2.1-\mathrm{mJ}$ fundamental pulses and $0.36-\mathrm{mJ}$ second harmonic pulses. Keeping the excitation parameters fixed, we increment the gas density from $n_{i}=1 \times 10^{14} \mathrm{~cm}^{-3}$ to $n_{i}=1.5 \times$ $10^{18} \mathrm{~cm}^{-3}$ and perform two simulations for each case: one with back-EMF included and the other without. In the absence of back-EMF, one would expect linear scaling of
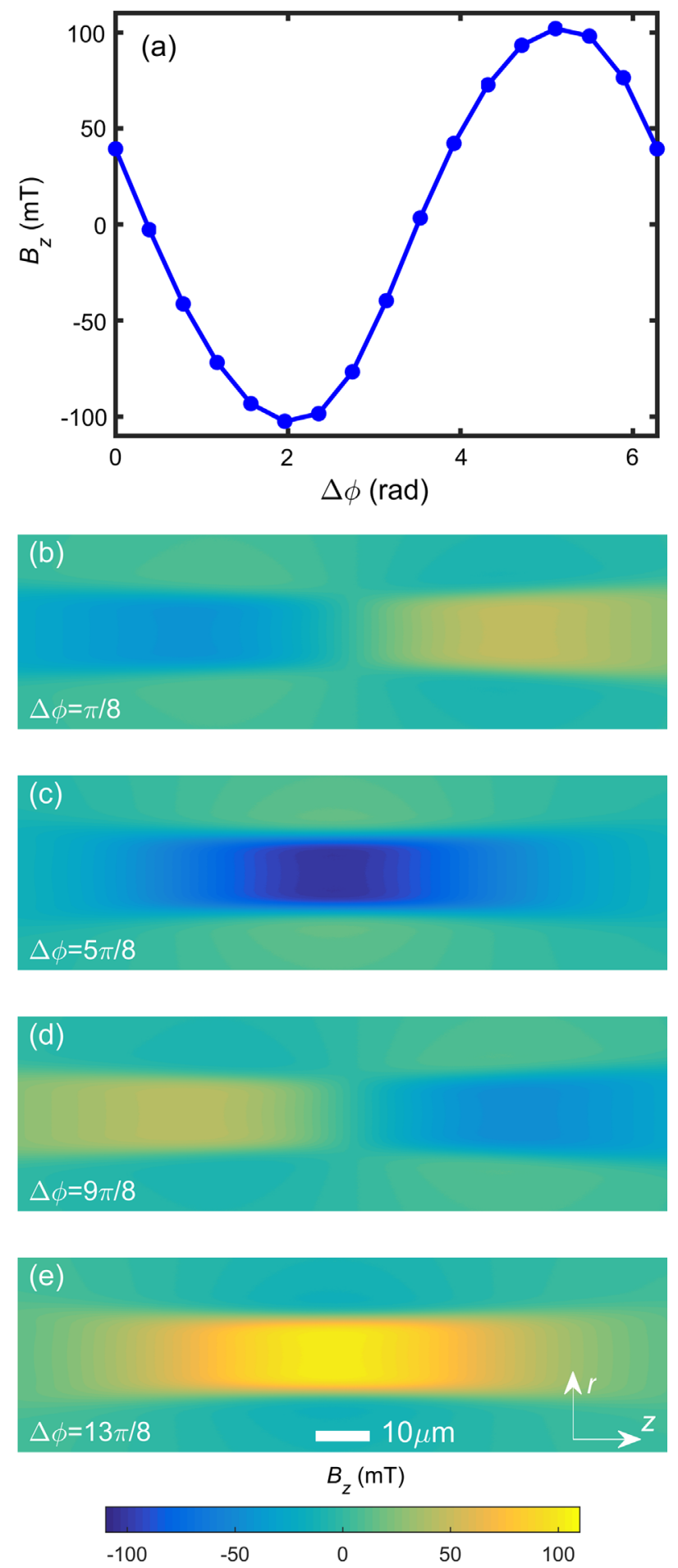

FIG. 3. Coherent control of magnetic fields. Simulation results for a beam waist $w_{0}=6 \mu \mathrm{m}$, fundamental wavelength $\lambda=4 \mu \mathrm{m}$, medium thickness $L=300 \mu \mathrm{m}$, equal $\omega / 2 \omega$ pulse durations $\tau_{p}=40 \mathrm{fs}$, and gas density $n_{i}=10^{17} \mathrm{~cm}^{-3}$. (a) Adjusting the phase offset between the $\omega$ and $2 \omega$ beams enables precise control of the direction and amplitude of the generated magnetic field. In addition, the relative phase influences the spatial distribution of the magnetic fields. Longitudinal slices of the magnetic fields generated in the focusing geometry are shown for (b) $\Delta \varphi=\pi / 8$, (c) $\Delta \varphi=5 \pi / 8$, (d) $\Delta \varphi=9 \pi / 8$, and (e) $\Delta \varphi=13 \pi / 8$. 

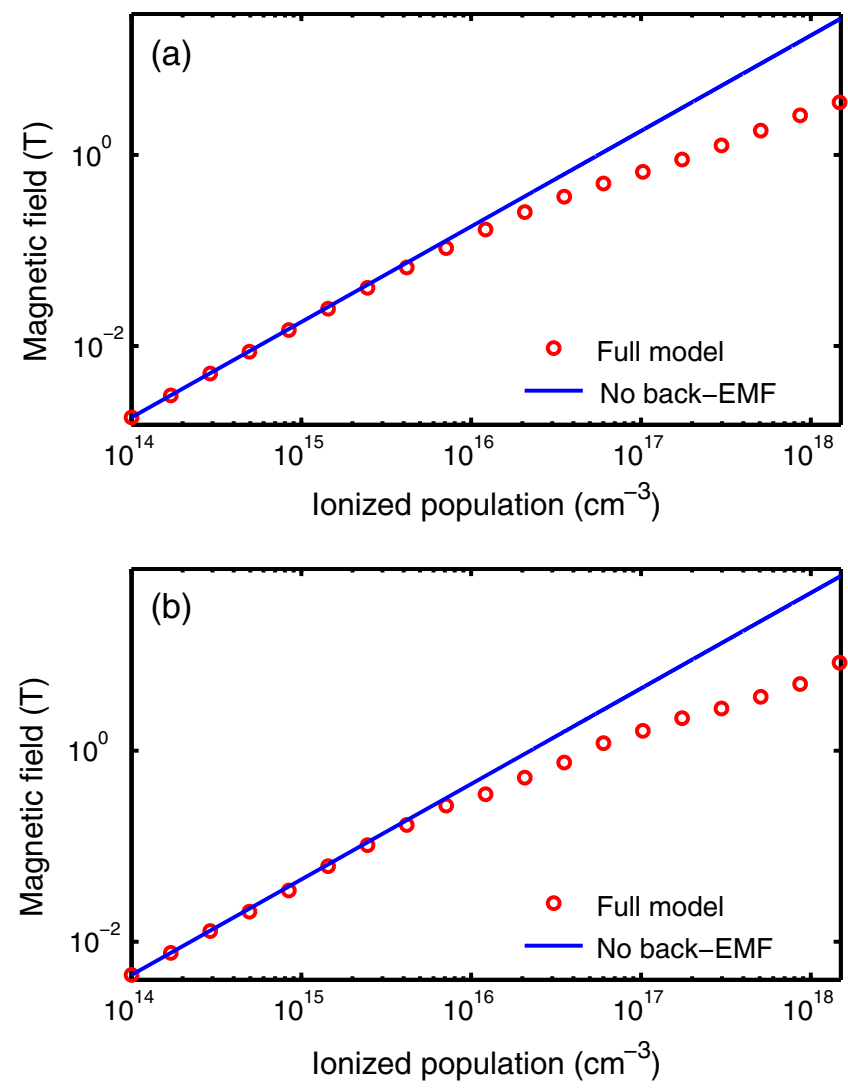

FIG. 4. The influence of back-EMF on magnetic field scaling. Simulation results for atomic hydrogen and helium, for a beam waist $w_{0}=100 \mu \mathrm{m}$, fundamental wavelength $\lambda=4 \mu \mathrm{m}$, medium thickness $L=150 \mu \mathrm{m}$, equal $\omega / 2 \omega$ pulse durations $\tau_{p}=40 \mathrm{fs}$, and variable gas density. For each gas density, one simulation is performed without back-EMF included, and another including back-EMF is performed. (a) Magnetic fields calculated for atomic hydrogen [fundamental peak electric field $E_{\omega}=2.9 \mathrm{~V} / \AA$ $\left(I_{\omega}=1.1 \times 10^{14} \mathrm{~W} / \mathrm{cm}^{2}\right)$, second harmonic peak electric field $\left.E_{2 \omega}=1.2 \mathrm{~V} / \AA\left(I_{2 \omega}=0.19 \times 10^{14} \mathrm{~W} / \mathrm{cm}^{2}\right)\right]$ reach a maximum amplitude of $B=3.6 \mathrm{~T}$ and are significantly suppressed by backEMF. (b) Because of a higher ionization potential, stronger electric fields [fundamental peak electric field $E_{\theta, \omega}=7.4 \mathrm{~V} / \AA$ $\left(I_{\omega}=7.3 \times 10^{14} \mathrm{~W} / \mathrm{cm}^{2}\right)$ and second harmonic peak electric field $\left.E_{\theta, 2 \omega}=2.3 \mathrm{~V} / \AA\left(I_{2 \omega}=0.70 \times 10^{14} \mathrm{~W} / \mathrm{cm}^{2}\right)\right]$ can be applied to atomic helium while maintaining the same ionization dynamics. This method counteracts back-EMF and enables the generation of magnetic fields up to $B=8.4 \mathrm{~T}$.

the current density and magnetic field with respect to the gas density.

The result of these simulations is shown in Fig. 4(a). Consistent with intuition gained from the ideal solenoid equation, magnetic field amplitudes up to $B_{z}=26.8 \mathrm{~T}$ are calculated in the absence of back-EMF. However, including back-EMF in the simulations as the gas density is increased beyond $10^{15} \mathrm{~cm}^{-3}$ is essential, as it limits the maximum magnetic field amplitude to $B_{z}=3.6 \mathrm{~T}$. As the gas density is increased, the backaction of the excited current density on the propagating electric fields becomes increasingly severe. This case results in the accumulation of a complicated phase to the exciting electric fields as they propagate, which strongly influences the coherent control process, the excited current density, and the resulting magnetic fields.

Using a gas species with a higher ionization potential provides a route to further magnetic field scaling while maintaining the same ionization dynamics. The higher electric field amplitude required to photo-ionize atoms subsequently accelerates electrons to higher velocities. To this end, we consider atomic helium, which has a first ionization potential $\left(I_{p}=24.6 \mathrm{eV}\right)$ significantly greater than that of hydrogen. We perform identical simulations for atomic helium, increasing the fundamental electric field strength to $E_{\theta, \omega}=7.4 \mathrm{~V} / \AA\left(I_{\omega}=7.3 \times 10^{14} \mathrm{~W} / \mathrm{cm}^{2}\right)$ and the second harmonic electric field strength to $E_{\theta, 2 \omega}=$ $2.3 \mathrm{~V} / \AA \quad\left(I_{2 \omega}=0.70 \times 10^{14} \mathrm{~W} / \mathrm{cm}^{2}\right)$, which maintains the same ionization dynamics as for hydrogen. The pulse energies required for these excitation parameters are $13.8 \mathrm{~mJ}$ for the fundamental and $1.3 \mathrm{~mJ}$ for the second harmonic.

The result of these simulations is shown in Fig. 4(b). As expected, the stronger driving electric fields enable further magnetic field scaling, and for an ionized population of $n_{i}=1.5 \times 10^{18} \mathrm{~cm}^{-3}$, we calculate the generation of magnetic field amplitudes up to $B_{z}=8.4 \mathrm{~T}$. Such intense magnetic fields are similar to those that are produced in superconducting electromagnets, but they provide the possibility for ultrafast temporal resolution of magneticfield-induced dynamics.

In addition, we note several variations of this scheme that will enable further scaling of the magnetic field amplitude. Cylindrical vector beams can now be produced with pulse duration of less than 15 fs [36], thereby allowing significantly faster rise-time fields. In the other extreme, implementing this approach using kilo-Joule-scale pulses at laser facilities would provide yet higher electric fields from driving higher currents, albeit with more complex ionization and plasma dynamics. We envisage that high-energy lasers will provide a route to picosecond magnetic field impulses with record-breaking magnetic field strengths.

Additionally, a variation of this experiment is possible whereby the coherent control is driven by a circularly polarized beam, which has been shown to produce highenergy, above-threshold ionization electrons [37], and a radially polarized orbital angular-momentum beam. This combination could enable a threefold increase in the magnetic field amplitude.

Lastly, a radial electric field component could be added to the laser beams. This component could be used to drive the ionized population towards the center of the beam, effectively reducing the radius of the excited current density. Since the magnetic field at the center of a wire loop is inversely proportional to the radius of the circulating current, i.e., $B=\mu_{0} I / 2 R$, this method would enhance the magnetic field strength. 
In summary, we have used well-understood physical principles, supported by a detailed model, to demonstrate an all-optical method to generate magnetic field impulses. The concepts we use are well tested, and the model is similar to those used for simulating $\mathrm{THz}$ field generation. We show solenoidal electric currents generated through strong field coherent control in atomic hydrogen and helium, where the azimuthal electric fields of the light drive an azimuthal current. The amplitude and direction of this current can be precisely controlled by changing the relative phase between the $\omega$ and $2 \omega$ light waves. Increasing the radius of the exciting beams increases the total solenoidal current and the resulting magnetic field amplitude. We predict magnetic fields up to $8.4 \mathrm{~T}$ with a 50 -fs rise time using loose focusing conditions, midinfrared laser pulses, and atomic helium as a target. These calculations demonstrate all-optical generation of spatially isolated magnetic fields with amplitudes that are similar to those available in magnetic field facilities.

Such fields would be a powerful new tool for condensed matter physics, and atomic and molecular physics, and could potentially be used for guiding charged particle beams for plasma physics or inertial confinement fusion. In contrast to conventional magnetic fields, which have been limited in bandwidth to $\mathrm{GHz}$ frequencies, this scheme provides a route to magneto-optical pump-probe measurements at $\mathrm{THz}$ frequencies. The introduction of short, intense magnetic impulses in the form of electromagnetic waves would also enable optical synchronization with sophisticated detection schemes, such as magneto-optical sampling, high-harmonic magnetic circular dichroism spectroscopy, or spin-polarized attosecond electron diffraction, enabling temporal resolution of magnetic-field-induced microscopic dynamics. Magnetic field metrology based on this ultrafast optical generation scheme will enable new frontiers in magnetism, particularly scaling to higher magnetic fields and resolution of femtosecond and attosecond magnetization dynamics.

\section{ACKNOWLEDGMENTS}

This research was supported by the Natural Sciences and Engineering Research Council of Canada (NSERC) Discovery Grant Program, the Canada Research Chairs program, the United States Defense Advanced Research Projects Agency ("Topological Excitations in Electronics" Agreement No. D18AC00011), and the United States Air Force Office of Scientific Research (Grant No. FA9550-161-0109).

[1] S. Blundell, Magnetism in Condensed Matter (Oxford University, New York, 2001).

[2] W. Gerlach and O. Stern, Der Experimentelle Nachweis der Richtungsquantelung im Magnetfeld, Z. Phys. 9, 349 (1922).
[3] E. Beaurepaire, J.-C. Merle, A. Daunois, and J.-Y. Bigot, Ultrafast Spin Dynamics in Ferromagnetic Nickel, Phys. Rev. Lett. 76, 4250 (1996).

[4] W. Meissner and R. Ochsenfeld, Ein Neuer Effekt Bei Eintritt der Supraleitfähigkeit, Naturwissenschaften 21, 787 (1933).

[5] J. E. Kunzler, E. Buehler, F. S. L. Hsu, and J. H. Wernick, Superconductivity in $\mathrm{Nb}_{3} \mathrm{Sn}$ at High Current Density in a Magnetic Field of 88 kgauss, Phys. Rev. Lett. 6, 89 (1961).

[6] P. Giraldo-Gallo et al., Scale-Invariant Magnetoresistance in a Cuprate Superconductor, Science 361, 479 (2018).

[7] E. J. Sie et al., An Ultrafast Symmetry Switch in a Weyl Semimetal, Nature (London) 565, 61 (2019).

[8] C. Zhang et al., Quantum Hall Effect Based on Weyl Orbits in $\mathrm{Cd}_{3} \mathrm{As}_{2}$, Nature (London) 565, 331 (2019).

[9] A. A. Zibrov, E. M. Spanton, H. Zhou, C. Kometter, T. Taniguchi, K. Watanabe, and A. F. Young, Even-Denominator Fractional Quantum Hall States at an Isospin Transition in Monolayer Graphene, Nat. Phys. 14, 930 (2018).

[10] E. G. Harris, Unstable Plasma Oscillations in a Magnetic Field, Phys. Rev. Lett. 2, 34 (1959).

[11] R. F. Post, R. E. Ellis, and F. C. Ford, Stable Confinement of a High-Temperature Plasma, Phys. Rev. Lett. 4, 166 (1960).

[12] R. F. Post and W. A. Perkins, Velocity-Space Plasma Instabilities Observed in a Mirror Machine, Phys. Rev. Lett. 6, 85 (1961).

[13] D. W. McRobbie, E. A. Moore, M. J. Graves, and M. R. Prince, MRI from Picture to Proton (Cambridge University Press, Cambridge, England, 2006).

[14] L. Mestel and L. Spitzer, Jr., Star Formation in Magnetic Dust Clouds, Mon. Not. R. Astron. Soc. 116, 503 (1956).

[15] J.C. Ørstedt, Experimenta Circa Effectum Conflictus Electrici in Acum Magneticam, J. Chem. Phys. 29, 275 (1820), [Experiments on the effect of a current of electricity on the magnetic needle, Ann. Philos. 16, 273 (1820)].

[16] J.-B. Biot and F. Savart, Note Sur le Magnétisme de la Pile de Volta, Ann. Chim. Phys. 15, 222 (1820), [Note on the magnetism of Volta's battery, Early Electromagnetics-The First Law of Circulation, edited by (Pregamon, New York, 1965), pp. 118-119].

[17] I. Tudosa, C. Stamm, A. B. Kashuba, F. King, H. C. Siegmann, J. Stöhr, G. Ju, B. Lu, and D. Weller, The Ultimate Speed of Magnetic Switching in Granular Recording Media, Nature (London) 428, 831 (2004).

[18] J. Deschamps, M. Fitaire, and M. Lagoutte, Inverse Faraday Effect in a Plasma, Phys. Rev. Lett. 25, 1330 (1970).

[19] A. Denoeud, L. Chopineau, A. Leblanc, and F. Queré, Interaction of Ultraintense Laser Vortices with Plasma Mirrors, Phys. Rev. Lett. 118, 033902 (2017).

[20] D. Wu and J.W. Wang, Magetostatic Amplifier with Tunable Maximum by Twisted-Light Plasma Interactions, Plasma Phys. Controlled Fusion 59, 095010 (2017).

[21] R. Nuter, P. Korneev, I. Thiele, and V. Tikhonchuk, Plasma Solenoid Driven by a Laser Beam Carrying an Orbital Angular Momentum, Phys. Rev. E 98, 033211 (2018).

[22] Y. Shi, J. Vieira, R. M. G. M. Trines, R. Bingham, B. F. Shen, and R. J. Kingham, Magnetic Field Generation in 
Plasma Waves Driven by Copropagating Intense Twisted Lasers, Phys. Rev. Lett. 121, 145002 (2018).

[23] M. Shapiro and P. Brumer, Quantum Control of Molecular Processes (John Wiley \& Sons, New York, 2012).

[24] E. Dupont, P. B. Corkum, H. C. Liu, M. Buchanan, and Z. R. Wasilewski, Phase-Controlled Currents in Semiconductors, Phys. Rev. Lett. 74, 3596 (1995).

[25] R. Atanasov, A. Haché, J. L. P. Hughes, H. M. van Driel, and J. E. Sipe, Coherent Control of Photocurrent Generation in Bulk Semiconductors, Phys. Rev. Lett. 76, 1703 (1996).

[26] Q. Zhan, Cylindrical Vector Beams: From Mathematical Concepts to Applications, Adv. Opt. Photonics 1, 1 (2009).

[27] J. M. Dawson, Particle Simulation of Plasmas, Rev. Mod. Phys. 55, 403 (1983).

[28] See Supplemental Material at http://link.aps.org/ supplemental/10.1103/PhysRevX.10.011063 for a description of the simulation technique used to model electromagnetic wave propagation, strong field ionization, electron acceleration, and plasma back-action on the propagating fields.

[29] M. Kreß, T. Löffler, S. Eden, M. Thomson, and H. G. Roskos, Terahertz-Pulse Generation by Photoionization of
Air with Laser Pulses Composed of Both Fundamental and Second-Harmonic Waves, Opt. Lett. 29, 1120 (2004).

[30] R. W. Hellwarth and P. Nouchi, Focused One-Cycle Electromagnetic Pulses, Phys. Rev. E 54, 889 (1996).

[31] D. H. Auston, K. P. Cheung, and P. R. Smith, Picosecond Photoconducting Hertzian Dipoles, Appl. Phys. Lett. 45, 284 (1984).

[32] Q. Wu and X.-C. Zhang, Free-Space Electro-Optic Sampling of Terahertz Beams, Appl. Phys. Lett. 67, 3523 (1995).

[33] E. Goulielmakis et al., Direct Measurement of Light Waves, Science 305, 1267 (2004).

[34] K. T. Kim, C. Zhang, A. D. Shiner, B. E. Schmidt, F. Légaré, D. M. Villeneuve, and P. B. Corkum, Petahertz Optical Oscilloscope, Nat. Photonics 7, 958 (2013).

[35] S. Keiber, S. Sederberg, A. Schwarz, M. Trubetskov, V. Pervak, F. Krausz, and N. Karpowicz, Electro-Optic Sampling of Near-Infrared Waveforms, Nat. Photonics 10, 159 (2016).

[36] F. Kong, H. Larocque, E. Karimi, P. B. Corkum, and C. Zhang, Generating Few-Cycle Radially Polarized Pulses, Optica 6, 160 (2019).

[37] P. B. Corkum, N. H. Burnett, and F. Brunel, Above-Threshold Ionization in the Long-Wavelength Limit, Phys. Rev. Lett. 62, 1259 (1989). 